\title{
RIPK3 contributes to TNFR1-mediated RIPK1 kinase-dependent apoptosis in conditions of clAP1/2 depletion or TAK1 kinase inhibition
}

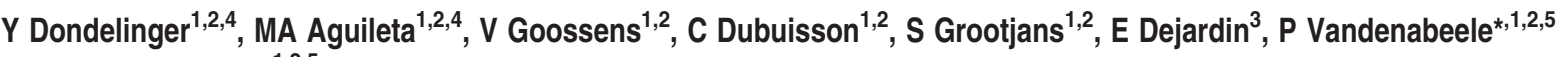 \\ and MJM Bertrand ${ }^{\star, 1,2,5}$
}

Receptor-interacting protein kinase (RIPK) 1 and RIPK3 have emerged as essential kinases mediating a regulated form of necrosis, known as necroptosis, that can be induced by tumor necrosis factor (TNF) signaling. As a consequence, inhibiting RIPK1 kinase activity and repressing RIPK3 expression levels have become commonly used approaches to estimate the contribution of necroptosis to specific phenotypes. Here, we report that RIPK1 kinase activity and RIPK3 also contribute to TNFinduced apoptosis in conditions of cellular inhibitor of apoptosis 1 and 2 (cIAP1/2) depletion or TGF- $\beta$-activated kinase 1 (TAK1) kinase inhibition, implying that inhibition of RIPK1 kinase activity or depletion of RIPK3 under cell death conditions is not always a prerequisite to conclude on the involvement of necroptosis. Moreover, we found that, contrary to cIAP1/2 depletion, TAK1 kinase inhibition induces assembly of the cytosolic RIPK1/Fas-associated protein with death domain/caspase-8 apoptotic TNF receptor 1 (TNFR1) complex llb without affecting the RIPK1 ubiquitylation status at the level of TNFR1 complex l. These results indicate that the recruitment of TAK1 to the ubiquitin $(\mathrm{Ub})$ chains, and not the $\mathrm{Ub}$ chains per se, regulates the contribution of RIPK1 to the apoptotic death trigger. In line with this, we found that cylindromatosis repression only provided protection to TNFmediated RIPK1-dependent apoptosis in condition of reduced RIPK1 ubiquitylation obtained by cIAP1/2 depletion but not upon TAK1 kinase inhibition, again arguing for a role of TAK1 in preventing RIPK1-dependent apoptosis downstream of RIPK1 ubiquitylation. Importantly, we found that this function of TAK1 was independent of its known role in canonical nuclear factor- $\kappa B$ $(\mathrm{NF}-\kappa \mathrm{B})$ activation. Our study therefore reports a new function of TAK1 in regulating an early NF- $\kappa \mathrm{B}$-independent cell death checkpoint in the TNFR1 apoptotic pathway. In both TNF-induced RIPK1 kinase-dependent apoptotic models, we found that RIPK3 contributes to full caspase-8 activation independently of its kinase activity or intact RHIM domain. In contrast, RIPK3 participates in caspase-8 activation by acting downstream of the cytosolic death complex assembly, possibly via reactive oxygen species generation.

Cell Death and Differentiation (2013) 20, 1381-1392; doi:10.1038/cdd.2013.94; published online 26 July 2013

Tumor necrosis factor (TNF) is a pleiotropic cytokine that controls a variety of cellular responses, including proliferation, differentiation, inflammatory cytokine production, survival and death. ${ }^{1}$ TNF signals by binding and activating two cell surface receptors, TNF receptor (TNFR) 1 and TNFR2, ${ }^{2}$ but most of its biological activities have been associated with TNFR $1 .{ }^{3}$ In most cell types, TNFR1 activation does not induce cell death but instead leads to the transcriptional upregulation of genes encoding pro-survival and pro-inflammatory molecules. ${ }^{4}$ This function is mediated by the assembly of a plasma membranebound multiprotein signaling complex, referred to as TNFR1 complex $\mathrm{I}^{5}$ that contains TNF receptor-associated death domain (TRADD), receptor-interacting protein kinase 1 (RIPK1, also known as RIP1), TNF receptor-associated factor 2, cellular inhibitor of apoptosis protein 1 and 2 (clAP1/2) and the linear ubiquitin (Ub) chain assembly complex. ${ }^{6}$ Within this complex, RIPK1 and other proteins are rapidly conjugated with Ub chains of various types. ${ }^{7-10}$ These Ub chains act as scaffolds for the recruitment and activation of the TAK1-binding protein $2 / 3$ (TAB2/3)-TGF- $\beta$ activated kinase 1 (TAK1) complex and the inhibitor of nuclear factor $-\kappa \mathrm{B}(\mathrm{NF}-\kappa \mathrm{B})$ kinase (IKK) complex (NEMO-IKK $\alpha-\mathrm{IKK} \beta)$,

\footnotetext{
1Department for Molecular Biomedical Research, VIB-Ghent University, Technologiepark 927, Zwijnaarde-Ghent, Belgium; ${ }^{2}$ Department of Biomedical Molecular Biology, Ghent University, Technologiepark 927, Zwijnaarde-Ghent, Belgium and ${ }^{3}$ Laboratory of Molecular Immunology and Signal Transduction, GIGA-Research, University of Liège, Liège, Belgium

${ }^{*}$ Corresponding author: P Vandenabeele or MJM Bertrand, Department for Molecular Biomedical Research, VIB-Ghent University, Technologiepark 927, Zwijnaarde-Ghent 9052, Belgium. Tel: + 320933 13722; Fax: + 320933 13609; E-mail: Peter.Vandenabeele @ dmbr.vib-UGent.be or Mathieu.Bertrand@dmbr.vib-UGent.be

${ }^{4}$ These authors contributed equally to this work.

${ }^{5}$ These two authors are co-senior authors.

Keywords: RIPK3; TAK1; CYLD; apoptosis; necroptosis; Nec-1

Abbreviations: RIPK1, receptor interacting protein kinase 1; clAP1/2, cellular inhibitor of apoptosis 1 and 2; CYLD, cylindromatosis; TNF, tumor necrosis factor; TNFR1, TNF receptor 1; TAK1, TGF- $\beta$-activated kinase 1; ROS, reactive oxygen species; TRADD, TNF receptor-associated death domain; TAB2/3, TAK1-binding protein 2/3; NF- $\kappa$ B, nuclear factor kappa B; IKK, inhibitor of nuclear factor kappa B kinase; CHX, cycloheximide; FADD, Fas-associated protein with death domain; SM, Smac Mimetic; Nec-1, necrostatin-1; zVAD-fmk, Z-Val-Ala-DL-Asp-fluoromethylketone; Ub, ubiquitin; BHA, butylated hydroxyanisole; KD, kinase-dead mutant Received 14.12.12; revised 15.5.13; accepted 25.6.13; Edited by G Melino; published online 26.7.13
} 
which subsequently leads to the activation of the mitogenactivated protein kinases and canonical NF- $\kappa$ B signaling pathways that collectively drive transcription of genes that prevent cell death and sustain inflammation. Accordingly, when the NF- $\kappa$ B response is inhibited, either genetically or by the use of the general translation inhibitor cycloheximide (CHX), TNFR1 ligation switches from a pro-survival to a pro-apoptotic response. This switch occurs via a mechanism that involves internalization of complex I and assembly of a cytoplasmic caspase-8-inducing death complex, known as TNFR1 complex Ila, which contains TRADD, Fas-associated protein with death domain (FADD) and procaspase-8..$^{5,11}$ Under these conditions, TNF-mediated death was shown not to depend on RIPK1. ${ }^{12,13}$

clAP1/2 are required for TNF-induced canonical NF- $\kappa$ B activation. ${ }^{10,14-16}$ Consequently, their depletion, obtained either genetically or by the use of Smac Mimetics (SM), also induces a switch to apoptosis. Intriguingly, in the absence of clAP1/2, TNF-induced death was shown to rely on RIPK1 and not on TRADD, ${ }^{12,15,17-19}$ suggesting that $\mathrm{ClAP} 1 / 2$ additionally regulate an $\mathrm{NF}-\kappa \mathrm{B}$-independent cell death checkpoint in the TNFR1 pathway. To discriminate the RIPK1-containing cytosolic death complex obtained in clAP1/2-depleted conditions from complex Ila, it has been defined as complex Ilb. ${ }^{11}$ The molecular mechanism accounting for the differential assembly of complex Ila versus $\mathrm{Ilb}$ is poorly understood, but suggested to rely on RIPK1 ubiquitylation status in complex I. Indeed, clAP1/2 act as direct ubiquitin ligases for $\mathrm{RIPK} 1^{8,14,15,20,21}$ and clAP1/2-mediated RIPK1 ubiquitylation in complex I is believed to prevent the transition of RIPK 1 from complex I to complex II. ${ }^{12,15,22,23}$ A notion supported by the fact that repression of the RIPK1 deubiquitinase cylindromatosis (CYLD) inhibits recruitment of RIPK1 to complex Ilb. ${ }^{12}$

In addition to apoptosis, TNF signaling can also induce necroptosis, a regulated form of necrosis that prevails in caspase-8-inhibited conditions, and whose physiological relevance has recently been demonstrated by in vivo studies. ${ }^{24}$ TNF-mediated necroptosis relies on the assembly of another cytosolic death complex, known as the necrosome, which consists of FADD, RIPK1 and RIPK3. ${ }^{25-27}$ Because both RIPK1 and RIPK3 kinase activities were shown to be crucial for necroptosis induction, necrostatin-1 (Nec-1), an inhibitor of RIPK1 kinase activity, ${ }^{28}$ is commonly used as a chemical tool to inhibit necroptosis. As an alternative approach, RIPK3 depletion has become a gold standard to estimate the contribution of necroptosis in a defined phenotype. ${ }^{29}$

In this study, we report on a new NF- $\kappa \mathrm{B}$-independent but kinase-dependent function of TAK1 in protecting cells from complex IIb-mediated apoptotic cell death. Because TAK1 kinase inhibition induces complex $\mathrm{Ilb}$ assembly without affecting RIPK1 ubiquitylation in complex I, our results suggest that the recruitment of TAK1 to the Ub chains conjugated to RIPK1 and not the Ub chains per se regulates the death potential of RIPK1. In addition, we demonstrate that the use of Nec-1 or the depletion of RIPK3 protects cells from TNF-mediated RIPK1-dependent apoptosis, both in conditions of clAP1/2 depletion or TAK1 kinase inhibition.

\section{Results}

TAK1 kinase activity protects cells from TNF-induced RIPK1 kinase-dependent apoptosis independently of NF- $\boldsymbol{k}$ B activation. TNFR1 engagement has been reported to mediate both RIPK1-independent and -dependent apoptosis based on the cellular context. ${ }^{12,15,17}$ The kinase activity of TAK1 has a crucial role in the activation of the mitogen-activated protein kinases and canonical NF- $\kappa \mathrm{B}$ pathways downstream of TNFR1. ${ }^{30,31}$ As a consequence, TAK1-deficient cells were shown to succumb by apoptosis upon single TNF stimulation. ${ }^{31-33}$ However, whether these cells die apoptotically in a RIPK1-independent or -dependent manner is unclear. Because TAK1 recruitment to complex I is reported to occur downstream of RIPK1 ubiquitylation, we speculated that TAK1 kinase inhibition would sensitize cells to TNF-induced apoptosis in a RIPK1-independent manner. Surprisingly, we found that RIPK1-deficiency protected immortalized MEFs against death induced by TNF in the presence of the TAK1 kinase inhibitor NP-009245 (TNF + TAK1i) similarly as against co-treatment with the SM $\mathrm{CmpA}^{34}$ (TNF + SM; Figures 1a and b, Supplementary Figures S1A, B). Of note, at the concentrations used, cell death occurred more rapidly with the TAK1 kinase inhibitor than with the SM. As negative control, we treated the MEFs with TNF in the presence of low concentration of $\mathrm{CHX}$ $(\mathrm{TNF}+\mathrm{CHX})$ and, as previously reported, ${ }^{13}$ observed that Ripk $^{-/-}$MEFs were not protected but sensitized to this apoptotic trigger (Supplementary Figure S2). Interestingly, we found that inhibiting RIPK1 kinase activity by Nec-1-protected Ripk1+/+ MEFs to either TNF + TAK1i or $\mathrm{TNF}+\mathrm{SM}$ as efficiently as the RIPK1 deficiency itself (Figures 1c and d, Supplementary Figures S1A and B). As RIPK1 kinase activity is mainly associated with necroptotic cell death, we confirmed protection to apoptosis, and not necroptosis, by measuring caspase-3 activity by DEVDase assay (Figures 1e-h, Supplementary Figures S1C and D, 3), by monitoring processing of caspase-8, caspase-3 and PARP (Figures $1 \mathrm{i}$ and $\mathrm{j}$ ) and by analyzing the cell death morphology (Supplementary Figure S4). Of note, although apoptosis induction upon TNF+TAK1i or TNF + SM treatment had been confirmed, we found that inhibiting caspase activity by the use of the pan caspase inhibitor zVAD-fmk (Z-Val-Ala-DL-Asp-fluoromethylketone) did not protect cells from death but instead induced a switch to RIPK1/3-dependent necroptosis in both conditions (Supplementary Figures S4, S5A and SB and data not shown). To exclude an off-target effect of the TAK1 kinase inhibitor, we confirmed the requirement of RIPK1 kinase activity for TNF-mediated apoptosis in the absence of functional TAK1 using Tak $1^{+/+}$and $T a k 1^{-/-}$MEFs treated with TNF in the absence or presence of Nec-1 (Supplementary Figure S5C). Of note, the TAK1 kinase inhibitor did not sensitize Tak $1^{-/-}$MEFs to apoptosis induced by TNF, demonstrating the TAK1-specific effect of the inhibitor (data not shown).

Finally, to further investigate whether the protective role of TAK1 and clAP1/2 against RIPK1-dependent apoptosis was depending on NF- $\kappa \mathrm{B}$ activation, we analyzed the effect of TAK1 inhibition or clAP1/2 depletion in MEFs unable to mount an NF- $\kappa \mathrm{B}$ response due to stable expression of an $I_{\kappa} \mathrm{B} \alpha$ 

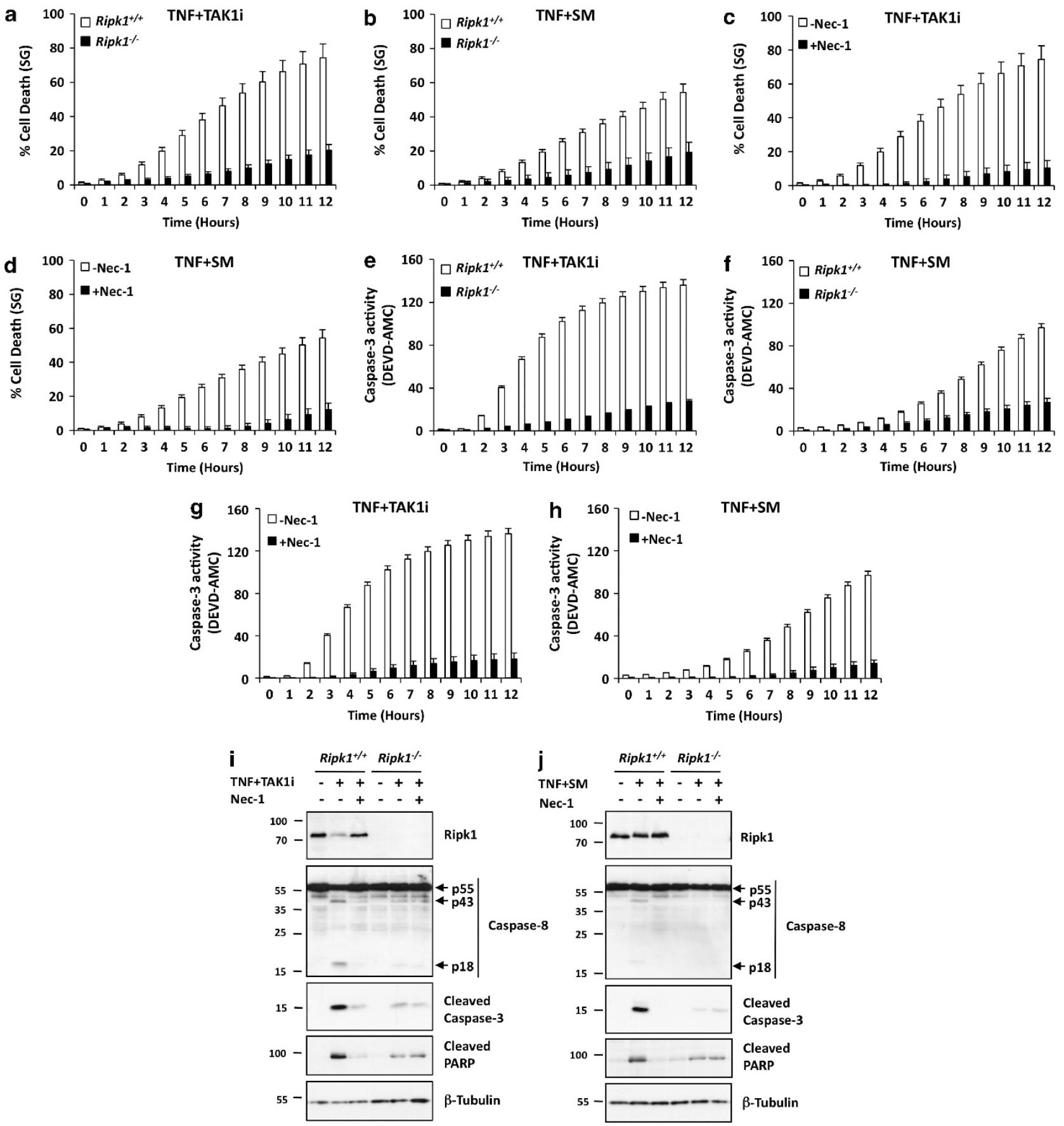

Figure 1 TAK1 kinase inhibition induces RIPK1 kinase-dependent apoptosis upon TNF stimulation. (a-h) Immortalized Ripk1 ${ }^{+/+}$and Ripk1 ${ }^{-/-}$MEFs were pre-treated for 30 min with TAK1i or SM in the presence or absence of Nec-1 and subsequently stimulated with hTNF. The percentage of cell death (SytoxGreen fluorescence) (a-d) and caspase-3 activity (DEVD-AMC fluorescence) (e-h) was calculated in function of time using the Fluostar Omega fluorescence plate reader as indicated in the experimental procedures. Error bars indicate the standard deviation from triplicate samples. The results are representative of at least three independent experiments. (i and j) Immortalized Ripk $1^{+/+}$and Ripk $1^{-/-}$MEFs were pre-treated for 30 min with TAK $1 \mathrm{i}$ (i) or SM (j) in the presence or absence of Nec-1 and subsequently stimulated with hTNF for $3 \mathrm{~h}$. Cells were then lysed and immunoblotted as indicated

mutant resistant to proteasomal degradation $\left(\left.\right|_{\kappa} \mathrm{B} \alpha \mathrm{SR}\right){ }^{35}$ As shown in Figure 2, single TNF stimulation induced RIPK1-kinase independent apoptosis in the $I_{\kappa} \mathrm{B} \alpha \mathrm{SR}$ MEFs, but co-stimulation with either TAK1i or SM led to an increase in cell death and caspase- 3 activity that could be inhibited by Nec-1.
Together, our results demonstrate that, similar to cIAP1/2 depletion by SM, TAK1 kinase inhibition induces RIPK1 kinase-dependent apoptosis upon TNF stimulation, which could be switched to necroptosis by inhibiting caspase activity. Importantly, the RIPK1 dependency in apoptosis induction originates from the fact that both TAK 1 and ClAP1/2 

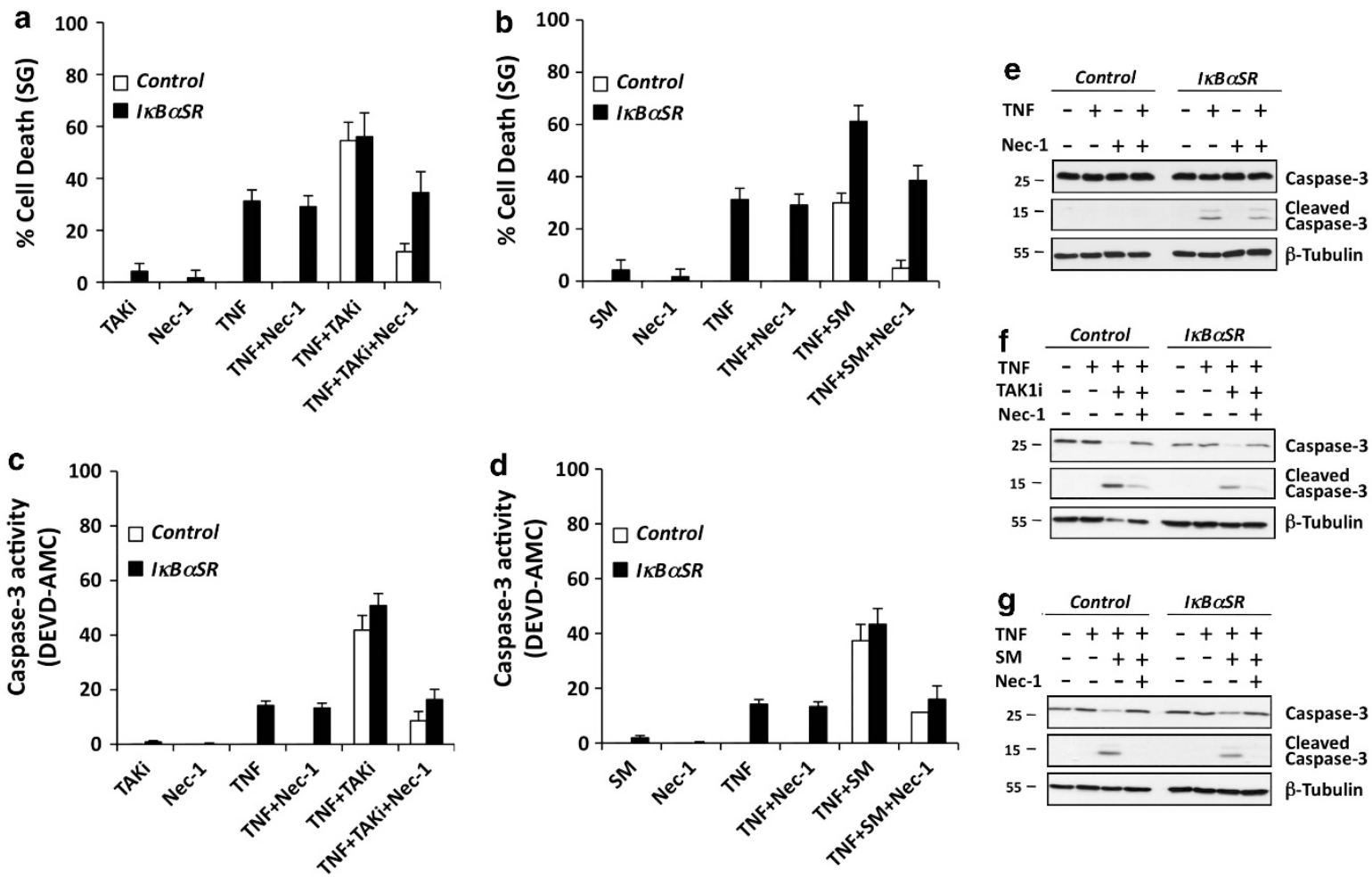

Figure 2 TAK1 and cIAP1/2 protect cells against TNF-mediated RIPK1-dependent apoptosis in a NF- $k$ B-independent manner. (a-d) Control MEFs or MEFs overexpressing $\mathrm{I}_{\kappa} \mathrm{B} \alpha \mathrm{SR}$ were pretreated with TAK1i (a and $\left.\mathbf{c}\right)$ or SM (b and $\left.\mathbf{d}\right)$ in the presence or absence of Nec-1 and subsequently stimulated with $\mathrm{hTNF}$ for $10 \mathrm{~h}$. Cell death $(\mathbf{a}$ and $\mathbf{~ b}$ ) and caspase-3 activity (c and $\mathbf{d}$ ) was analyzed at the indicated time using the Fluostar Omega fluorometer. Error bars indicate the standard deviation from triplicate samples. The results are representative of at least two independent experiments. $(\mathbf{e}-\mathbf{g})$ Control MEFs or MEFs overexpressing $\mid \kappa B \alpha S R$ were pretreated with media (e), TAK1i (f) or SM (g) in the presence or absence of Nec-1 and subsequently stimulated with hTNF for, respectively, $4 \mathrm{~h}(\mathbf{e})$ or $2 \mathrm{~h}$ (f and $\mathbf{g}$ ). Cells were then lysed and immunoblotted as indicated

regulate an early NF- $\kappa \mathrm{B}$-independent cell death checkpoint in the TNFR1 pathway, in addition to their reported role in $\mathrm{NF}-\kappa \mathrm{B}$ activation.

TAK1 kinase inhibition induces complex Ilb assembly without affecting RIPK1 ubiquitylation at complex I. The combination of TNF and SM leads to apoptosis through assembly of a caspase-8-activating complex, referred to as complex Ilb, consisting of FADD, procaspase-8 and RIPK1. ${ }^{12,15,17}$ To determine whether TAK1 kinase inhibition similarly induces TNF-mediated RIPK1-dependent apoptosis via complex Ilb assembly, we immunoprecipitated caspase-8 in MEFs stimulated either with TNF + TAK1i or TNF + SM, and analyzed RIPK1 recruitment by immunoblotting. These immunoprecipitations were performed in the absence or presence of zVAD-fmk, as previous studies indicated that complex Ilb detection requires caspase inhibition to avoid proteolytic cleavage of RIPK1 by capase-8. ${ }^{12,15,17,36}$ As shown in Figures $3 a$ and $b$, both TNF + TAK1i and TNF + SM treatments led to complex Ilb assembly, which was better detected in the presence of zVAD-fmk. Cleaved fragments of RIPK1 were mostly observed in the absence of zVAD-fmk. Importantly, Nec-1 completely inhibited complex Ilb assembly both in the absence or presence of zVAD-fmk, further demonstrating the kinase-dependent role of RIPK1 in TNF + TAK1i and TNF + SM apoptotic settings.

The recruitment of RIPK1 to complex Ilb in clAP1/2depleted conditions is thought to originate from defective
RIPK1 ubiquitylation at complex I. We therefore compared RIPK1 ubiquitylation status at the receptor complex by immunoprecipitating TNFR1 using FLAG-hTNF in MEFs pre-treated, or not, with either TAK1 inhibitor or SM for $30 \mathrm{~min}$. Although SM pre-treatment greatly affected RIPK1 ubiquitylation, TAK1 kinase inhibition had no effect on RIPK1 ubiquitylation status or on clAP1 levels (Figures $3 c$ and d). Of note, no difference in RIPK1 ubiquitylation levels was observed at the level complex Ilb between TNF + TAK1 $\mathrm{i}$ and $\mathrm{TNF}+\mathrm{SM}$ treatments (Figure $3 e$ ). Also, contrary to its crucial role in complex Ilb assembly, we found that RIPK1 kinase activity was dispensable for the recruitment and ubiquitylation of RIPK1 at complex I (Figure 3f).

Because the de-ubiquitinase CYLD was suggested to promote complex Ilb assembly under SM conditions by dismantling the Ub chains remaining on RIPK1 at complex I, ${ }^{12}$ we next compared the effect of CYLD repression on apoptotic cell death induced by TNF + TAK1i and TNF + SM. As shown in Figures 4a-d (and Supplementary Figure S6), CYLD repression provided protection to apoptosis induced by TNF + SM, but not by TNF+TAK1i. Those effects, respectively, correlated with defective and unaffected complex Ilb formation (Figure 4e).

Our data therefore indicate that it is not per se the Ub chains conjugated to RIPK1 at complex I but instead the recruitment of TAK1 to these Ub chains that, either directly or indirectly, regulates the integration of RIPK1 in complex Ilb. The fact that CYLD repression does not provide protection under TAK1 
a TNF -+-+++

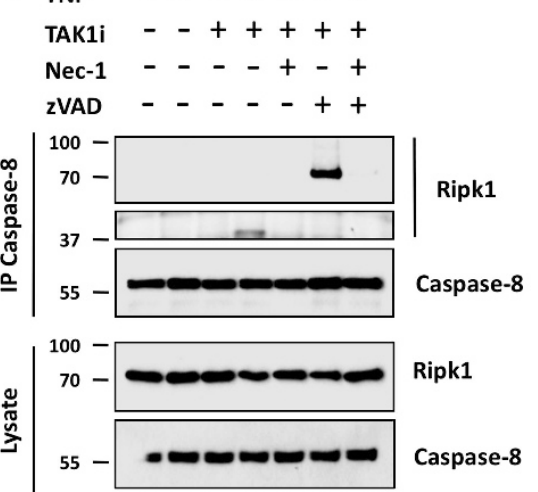

b TNF -+-+++

SM $\quad-+++++$

Nec-1 - - - + - +

zVAD - - - - +

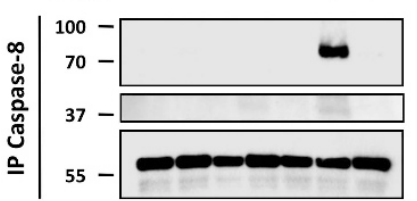

Ripk1

Caspase-8

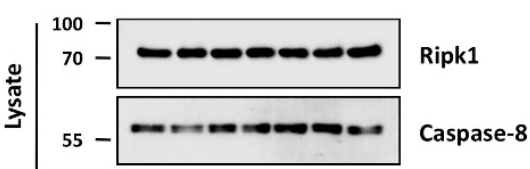

C FLAG-TNF $0 \begin{array}{llll}0 & 5 & 0 & 5\end{array}$

TAK1i - ++
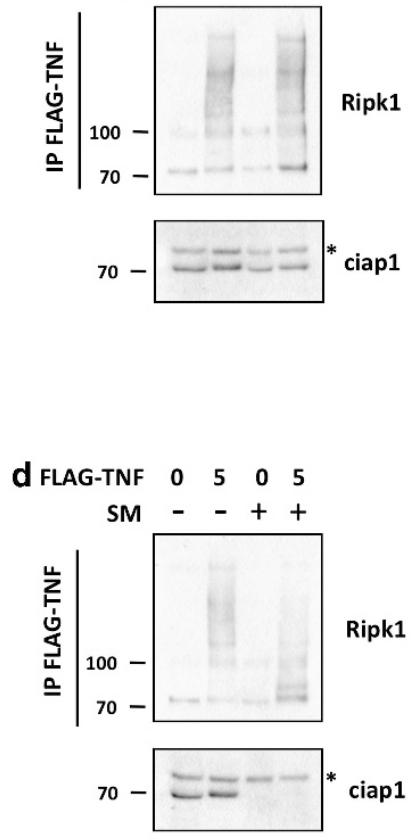

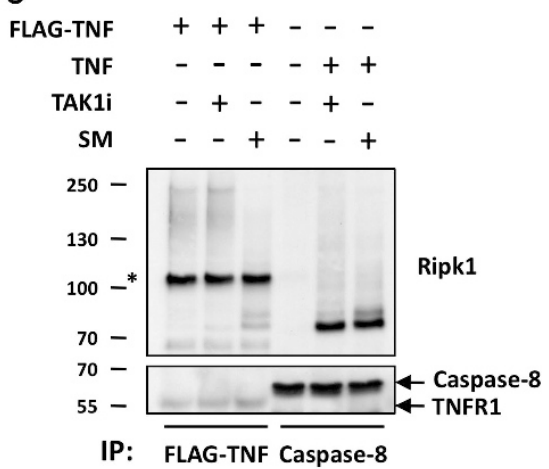

f FLAG-TNF -+++++

Nec-1 - +-+-+

TAK1i - -++-

SM - - - + +

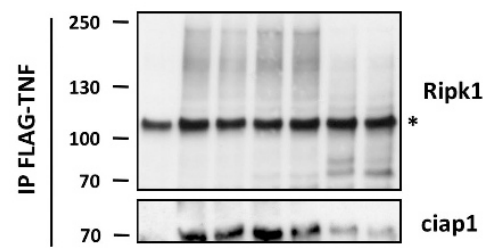

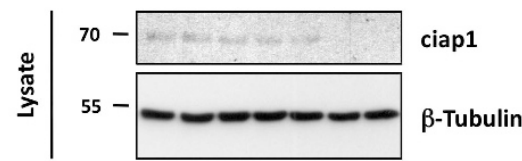

Figure 3 TAK1 kinase inhibition induces complex IIb assembly without affecting RIPK1 ubiquitylation at complex I. (a and b) Immortalized Ripk1 ${ }^{+/+}$MEFs were pretreated for 30 min with the indicated compounds in combination with TAK1i (a) or SM (b), followed by stimulation with hTNF for $2 \mathrm{~h}$. Next, complex II was isolated by immunoprecipitation of caspase-8 and analyzed by immunoblotting. (c and d) Immortalized Ripk $1^{+/+}$MEFs were pre-treated for 30 min with TAK1i (c) or SM (d) and subsequently stimulated for $5 \mathrm{~min}$ with FLAG-hTNF. Complex I was immunoprecipitated and the ubiquitylation status of TNFR1-bound RIPK1 was analyzed by immunoblotting. The levels of cIAP1 in the lysates are also shown. The asterisk shows a non-specific band recognized by the anti-clAP1 antibody. (e) Immortalized Ripk1+/+ MEFs were pretreated with TAK1i or SM and stimulated with either FLAG-hTNF for 5 min or hTNF for $2 \mathrm{~h}$. Ubiquitylation status of RIP1 in the different complexes was evaluated by immunoblotting after immunoprecipitation of FLAG-hTNF for complex I or caspase-8 for complex II. (f) Immortalized Ripk $1^{+/+}$MEFs were pre-treated for 30 min with TAK1i or SM in the presence or absence of Nec-1 and subsequently stimulated with FLAG-hTNF for 5 min. Complex I was immunoprecipitated and recruitment of RIPK1 to TNFR1 was analyzed by immunoblotting

kinase inhibition highlights the role of TAK1 downstream of RIPK1 ubiquitylation in the early NF- $\kappa$ B-independent cell death checkpoint of the TNFR1 pathway.

RIPK3 deficiency affects TNFR1-mediated RIPK1 kinase-dependent apoptosis. RIPK3 has emerged as an essential kinase in necroptosis induction ${ }^{25-27}$ and, as a consequence, RIPK3 repression has become a proof of principle to highlight the contribution of necroptosis in a defined phenotype; although initial reports on RIPK3 indicated that its ectopic expression could also induce apoptosis. $^{37,38}$ To investigate whether RIPK3 could contribute to TNF-induced RIPK1-dependent apoptosis, we stimulated immortalized Ripk3 ${ }^{+/+}$and Ripk3 ${ }^{-/-}$MEFs with TNF + SM and TNF + TAK1i. Remarkably, we observed that RIPK3 deficiency provided partial protection to both apoptotic triggers (Figures 5a-d, Supplementary Figure S7). The reduction in RIPK1-dependent apoptosis was accompanied by a reduction in caspase-8, caspase- 3 and PARP cleavage (Figures $5 e$ and f), indicating a role for RIPK3 upstream or at the level of caspase-8. To further validate the role of RIPK3 in TNFR1-mediated RIPK1-dependent caspase-8 activation, we immunoprecipitated active caspase-8 using biotin-IETDfmk in Ripk $3^{+/+}$and Ripk $3^{-/-}$primary MEFs stimulated with $\mathrm{TNF}+\mathrm{SM}$, and again observed defective caspase-8 activation in the absence of RIPK3 (Figure $5 \mathrm{~g}$ ). The role of RIPK3 in TNF + TAK1i- and TNF + SM-induced apoptosis was also confirmed by repressing RIPK3 in the Ripk $1^{+/+}$ MEFs using miRNA (Figures 5h-l, Supplementary Figure S8). Interestingly, RIPK3 deficiency did not provide protection to RIPK1-independent apoptosis induced by Fas ligation (Supplementary Figure S9).

Our results therefore demonstrate that RIPK3 specifically contributes to TNFR1-mediated RIPK1-dependent apoptosis, in addition to its well-established role in TNF-induced necroptosis.

RIPK3 differentially regulates TNF-mediated apoptosis and necroptosis. Necroptosis induction relies on both RIPK1 and RIPK3 kinase activities, and on the physical 
a
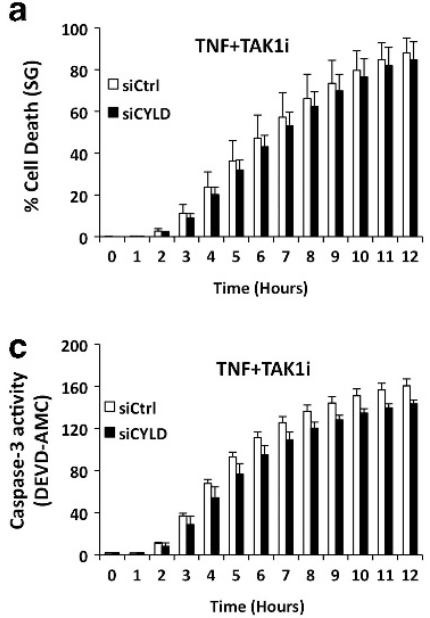

b
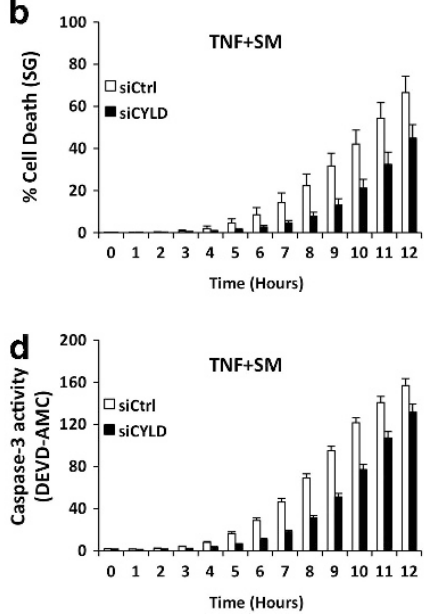
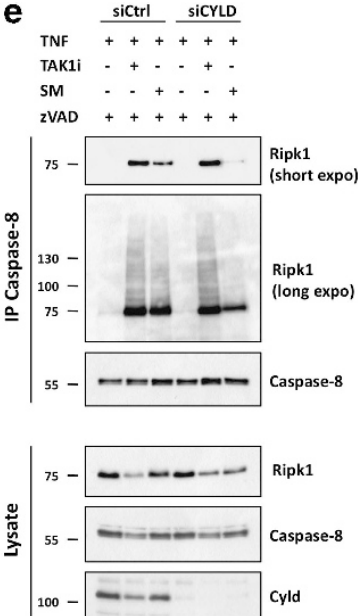

Figure 4 CYLD knockdown protects cells against RIPK1-dependent apoptosis induced by TNF and SM, but not by TNF and TAK1i. (a-e) Immortalized Ripk1+/+ MEFs were transfected with a control siRNA or siRNA-targeting CYLD. After $72 \mathrm{~h}$, cells were pretreated with TAK1i (a and $\mathbf{c}$ ) or SM (b and $\mathbf{d}$ ) for 30 min followed by stimulation with hTNF. Cell death ( $(\mathbf{a}$ and $\mathbf{b})$ and caspase-3 activity ( $\mathbf{c}$ and $\mathbf{d}$ ) were analyzed at the indicated time points using the Fluostar Omega fluorometer. Error bars indicate the standard deviation from triplicate samples. The results are representative of at least two independent experiments. (e) Immortalized Ripk1 ${ }^{+/+}$MEFs were transfected with a control siRNA or siRNA-targeting CYLD. After $72 \mathrm{~h}$, cells were pretreated with TAK1i or SM in the presence of ZVAD for 30 min followed by the stimulation with hTNF for $2 \mathrm{~h}$. Complex II assembly was analyzed by immunoblotting after immunoprecipitation of caspase-8

interaction between RIPK1 and RIPK3 via their respective RHIM domains. ${ }^{25,26}$ To test whether these domains of RIPK3 are also required for its contribution to TNF-mediated RIPK1dependent apoptosis, we lentivirally reconstituted Ripk3 $3^{-/-}$ MEFs with an empty vector (ctrl), with wild-type (WT) RIPK3 and with two mutants of RIPK3 reported to inhibit necroptosis induction: a kinase-dead mutant (KD) ${ }^{25}$ and a RHIM domain mutant $\left(\mathrm{RHIM}^{*}\right) .{ }^{26}$ Interestingly, we found that reconstitution of Ripk3 ${ }^{-1-}$ MEFs with all three RIPK3 variants equally rescued caspase-3 activation upon TNF + SM or TNF + TAK1i treatment (Figures $6 a$ and $b$ ). The extent of rescue correlated with the relative RIPK3 expression levels between the Ripk3 ${ }^{-/-}$reconstituted cells and the Ripk3 ${ }^{+/+}$MEFs (Figure 6c). Nevertheless, we observed that the cells reconstituted with WT RIPK3 succumbed to the treatment almost as efficiently as the Ripk $3^{+/+}$MEFs, whereas the cells reconstituted with $\mathrm{KD}$ and $\mathrm{RHIM}^{\star}$, RIPK3 only partially recovered the cell death sensitivity (Figure $6 \mathrm{~d}$ ). Because cells reconstituted with the two RIPK3 mutants are unable to rescue any sensitivity to RIPK3-dependent necroptosis (Supplementary Figure S10), the difference in cell death sensitivity between the three RIPK3 variants upon TNF + TAK1i treatment presumably originates from the fact that the ectopic expression of WT RIPK3 in Ripk3 ${ }^{-/-}$MEFs induces necroptosis on top of apoptosis, which is impossible for the two RIPK3 mutants.

Together, our data indicate that RIPK3 contributes to TNF-mediated RIPK1-dependent apoptosis independently of its kinase activity or intact RHIM, and therefore demonstrate that RIPK3 differentially regulates apoptosis and necroptosis downstream of TNFR1.

Reactive oxygen species (ROS) scavenging inhibits TNFR1-mediated RIPK1-dependent apoptosis. It was recently suggested that, in the context of the inflammasome, RIPK3 induces caspase-8 activation through ROS production via an uncharacterized mechanism. ${ }^{39}$ To investigate whether the contribution of RIPK3 to caspase-8 activation in our cellular system also involves ROS generation, we first analyzed the effect of ROS scavenging, using butylated hydroxyanisole (BHA) and $n$-acetylcysteine, on RIPK1-dependent apoptosis induced by TNF + TAK1i and TNF + SM. We found that ROS scavenging protected the MEFs from these apoptotic triggers even more potently than Nec-1 (Figure 7a). Importantly, this protection was associated with the inhibition of caspase-8 and caspase-3 processing (Figures $7 b-d$ ), implying a role for ROS in the early signaling phase of RIPK1-dependent apoptosis. We therefore analyzed whether ROS scavenging could interfere with complex Ilb assembly, and found that $30 \mathrm{~min}$ pretreatment with BHA almost completely inhibited the association of RIPK1 with caspase-8 (Figure 7e). Of note, BHA pretreatment had no effects on the recruitment of RIPK1 to complex I (Figure 7f), demonstrating a role for ROS in the transition from complex I to complex Ilb.

RIPK3 regulates TNF-mediated RIPK1-dependent apoptosis downstream of complex IIb assembly, possibly via ROS generation. Knowing that caspase-8 activation was positively regulated by both RIPK3 and ROS in our TNF + TAK1i and TNF + SM settings, and that a RIPK3-ROScaspase- 8 activation axis had previously been suggested, ${ }^{39}$ we next analyzed the effect of RIPK3 deficiency on ROS generation upon TNF + TAK1i treatment. As RIPK3 repression is known to affect ROS levels under necroptotic conditions, ${ }^{40}$ we included TNF + TAK $1 \mathrm{i}+\mathrm{zVAD}$-fmk treatment as a positive control in our experiments. Remarkably, we found that RIPK3 deficiency affected ROS levels in both necroptotic and apoptotic conditions, whereas no difference in ROS levels was detected in unstimulated conditions (Figure 7g). However, and contrary to ROS scavenging, we found that RIPK3 deficiency did not affect complex IIb 

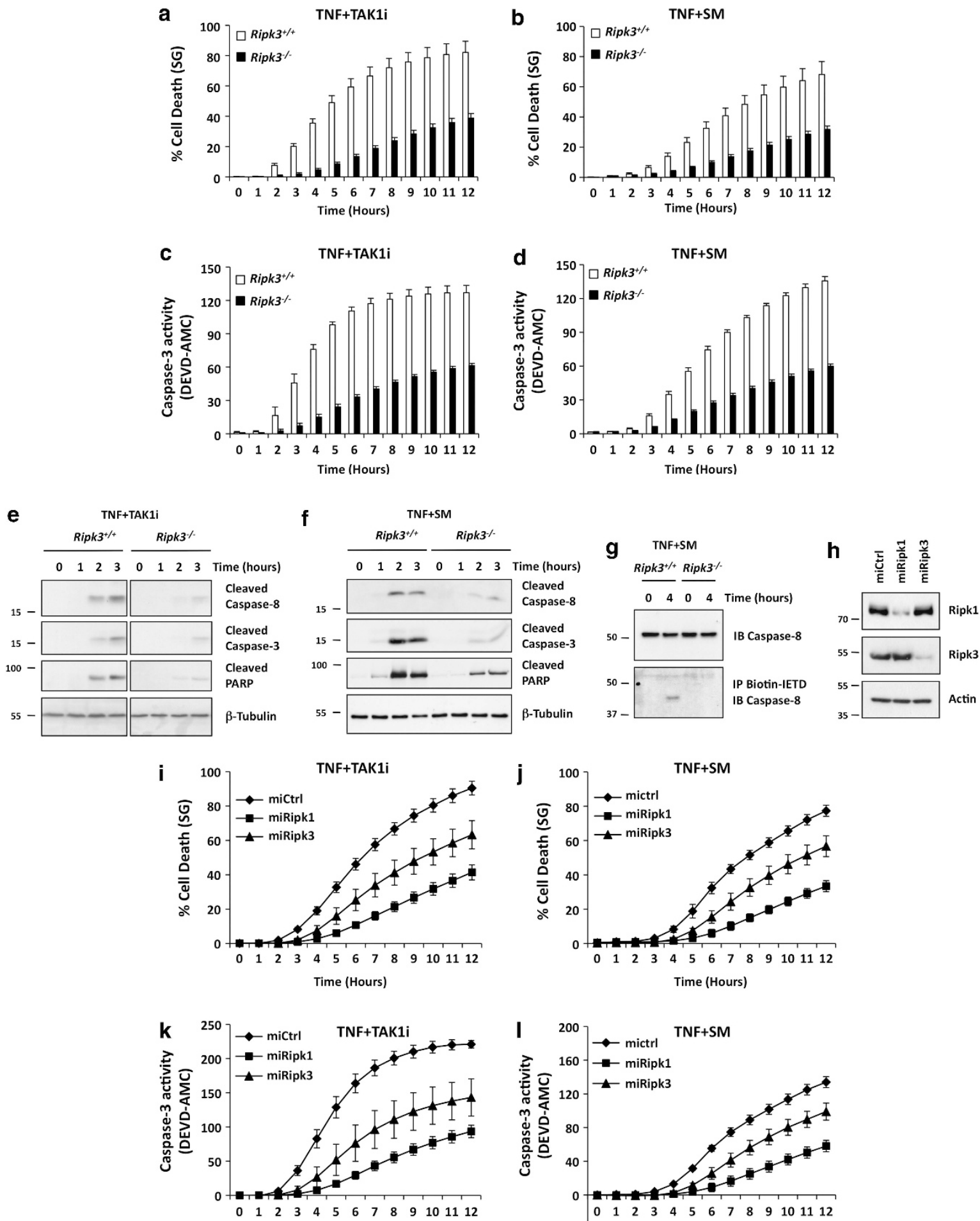

Figure 5 RIPK3 deficiency affects TNFR1-mediated RIPK1-dependent apoptosis. (a-d) Immortalized Ripk3 $3^{+/+}$and Ripk3 ${ }^{-/-}$MEFs were pre-treated for 30 min with TAK1i ( $\mathbf{a}$ and $\mathbf{c}$ ) or SM ( $\mathbf{b}$ and $\mathbf{d}$ ) and subsequently stimulated with hTNF. Cell death ( $\mathbf{a}$ and $\mathbf{b})$ and caspase-3 activity ( $\mathbf{c}$ and $\mathbf{d})$ were analyzed at the indicated time points using the Fluostar Omega fluorometer. Error bars indicate the standard deviation from triplicate samples. The results are representative of at least three independent experiments. (e and $\mathrm{f}$ ) Immortalized Ripk3 ${ }^{+/+}$and Ripk3 ${ }^{-/-}$MEFs were pre-treated for 30 min with SM (e) or TAK1i (f), followed by stimulation with hTNF for the indicated periods of time. Cells were

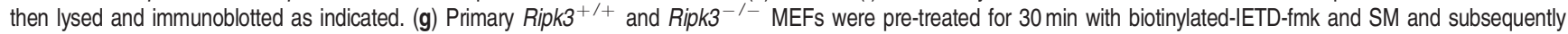
stimulated with hTNF for $4 \mathrm{~h}$. Cells were then lysed and caspase-8 bound to biotinylated-IETD-fmk was immunoprecipitated. The levels of pro-caspase-8 in the lysates and of cleaved-caspase-8 in the immunoprecipitate were then revealed by immunoblotting. (h-l) Immortalized Ripk1 ${ }^{+/+}$MEFs were lentivirally transduced with either a control miRNA or a miRNA-targeting RIPK1 or RIPK3. (h) Repression of RIPK1 or RIPK3 protein levels was revealed by immunoblotting. (i-I) miRNA-transduced Ripk1 ${ }^{+/+}$MEFs were pre-treated for 30 min with TAK1i ( $\mathbf{i}$ and $\mathbf{k}$ ) or SM ( $\mathbf{j}$ and $\mathbf{I})$ and subsequently stimulated with hTNF. Cell death (i and $\mathbf{j})$ or caspase-3 activity (k and $\mathbf{I}$ ) was analyzed at the indicated time points using the Fluostar Omega fluorometer. Error bars indicate the standard deviation from triplicate samples. The results are representative of at least two independent experiments 

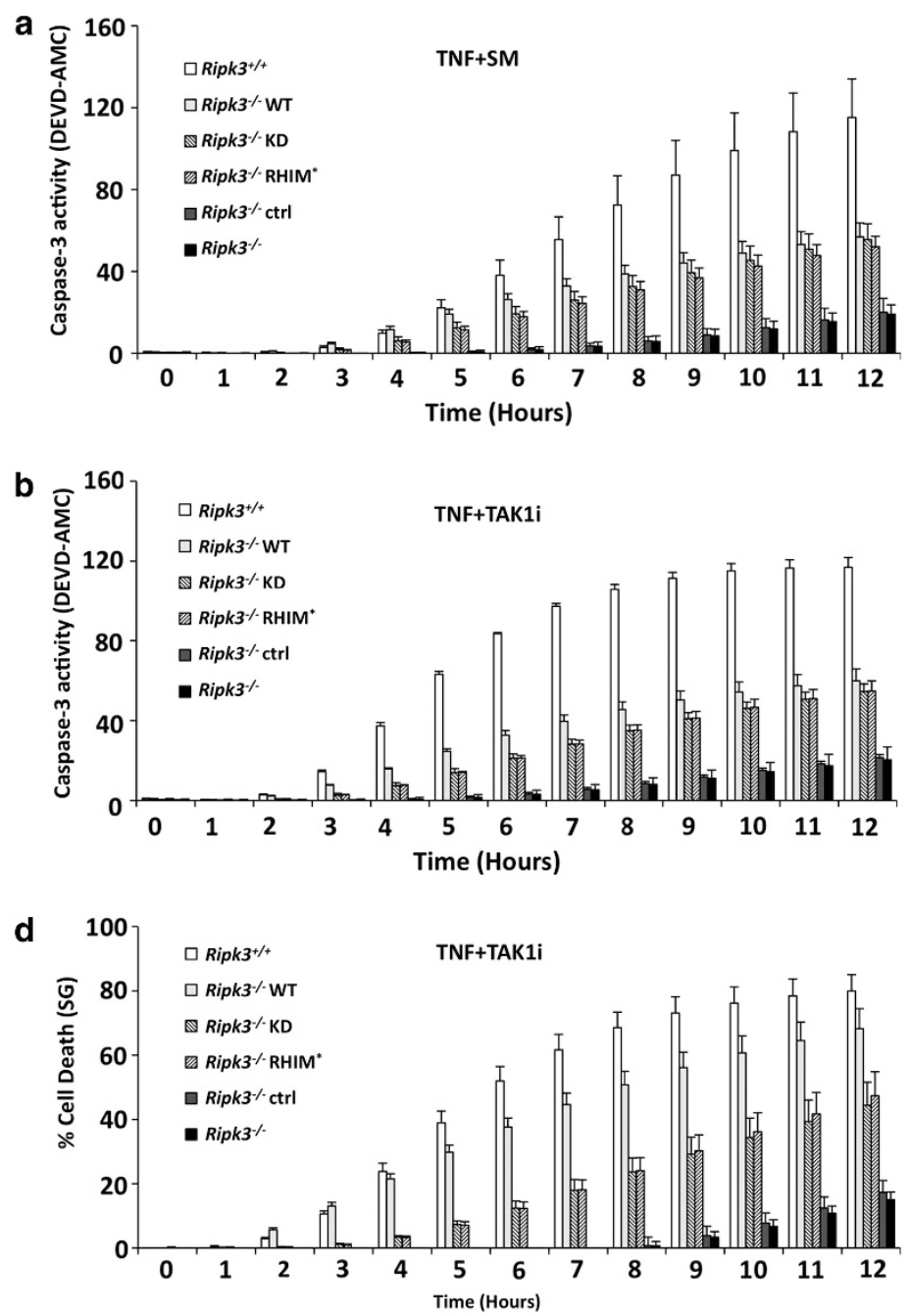

Figure 6 The kinase activity of RIPK3 and its intact RHIM domain are dispensable for TNFR1-mediated RIPK1-dependent apoptosis. (a-d) Immortalized Ripk3 ${ }^{-/-}$ MEFs were lentivirally reconstituted with an empty control vector (CTRL), wild-type Ripk3 (WT), kinase-dead Ripk3 (KD) or RHIM mutated Ripk3 (RHIM*). (a-d) The reconstituted Ripk3 $3^{-1-}$ MEFs were pre-treated for 30 min with SM (a) or TAK1i (b) and subsequently stimulated with hTNF. Caspase-3 activity (a and $\left.\mathbf{b}\right)$ and cell death (d) were analyzed at the indicated time points using the Fluostar Omega fluorometer. Error bars indicate the standard deviation from triplicate samples. The results are representative of at least three independent experiments. (c) RIPK3 expression levels in Ripk3 ${ }^{+/+}$and reconstituted Ripk3 ${ }^{-/-}$MEFs were compared by immunoblotting

assembly (Figure $7 \mathrm{~h}$ ), suggesting a role for RIPK3 in TNF-mediated RIPK1-dependent apoptosis downstream of complex IIb assembly, possibly via ROS generation.

Together, our data therefore demonstrate that ROS production is required upstream and possibly downstream of complex IIb assembly for full caspase-8 activation, and that RIPK3 only contributes to TNF-mediated RIPK1-dependent apoptosis downstream of complex IIb assembly, possibly via ROS generation (Figure 8).

\section{Discussion}

Depending on the cellular context, TNF can induce RIPK1independent or -dependent apoptosis by assembly of two different cytosolic death complexes, respectively, complex Ila or IIb. ${ }^{11}$ RIPK1-independent apoptosis is classically obtained by the addition of transcriptional or translational inhibitors, whereas RIPK1-dependent apoptosis is mostly observed in conditions of clAP1/2 depletion, obtained either by the use of SM or more physiologically after stimulation with TWEAK, which induces clAP1/2 degradation upon binding to its cognate receptor Fn14. ${ }^{12,15,17,21,41}$ As both approaches interfere with the canonical NF- $\kappa \mathrm{B}$-mediated pro-survival response, it is believed that the decision to undergo either complex Ila or IIb apoptosis is regulated at a different level. For instance, the Ub chains conjugated to RIPK1 by clAP1/2 at the receptor complex I are thought to constitute the decisive factor preventing RIPK1 from integrating complex II and limiting its contribution to the apoptotic dismantlement of the cell. $^{23}$ clAP1/2-mediated ubiquitylation of RIPK1, and potentially of other proteins within complex I, provides docking sites for the recruitment of the TAB-TAK1 complex. ${ }^{15,18,42}$ Here, we show that inhibition of TAK1 kinase activity triggers TNF-mediated complex Ilb-dependent apoptosis independently of NF- $\kappa$ B and without affecting ubiquitylation of RIPK1 at complex I, therefore demonstrating that the recruitment of TAK1 to the Ub chains, and not the Ub chains themselves, regulates the death potential of RIPK1. Consistently, CYLD 

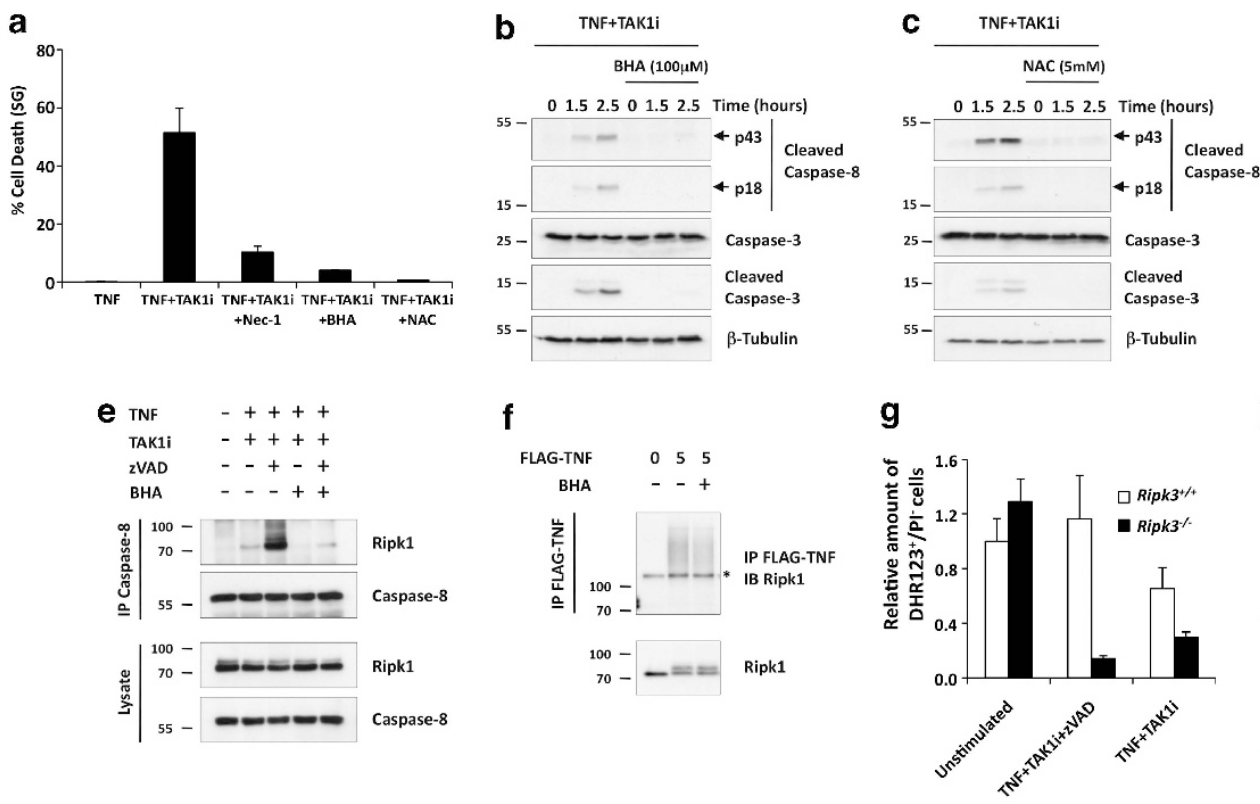
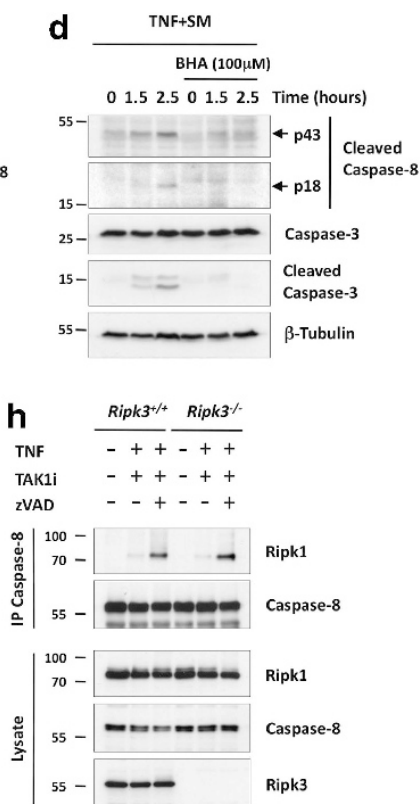

Figure 7 ROS scavenging inhibits TNFR1-mediated RIPK1-dependent apoptosis. (a) Immortalized Ripk1 $1^{+/+}$MEFs were pre-treated for 30 min with TAK1i in the presence of Nec-1, BHA or NAC and subsequently stimulated with hTNF for $4 \mathrm{~h}$. Cell death was analyzed using the Fluostar Omega fluorescence plate reader. Error bars indicate standard deviation from triplicate samples. Experiments are representative of at least three independent experiments. (b-d) Immortalized Ripk $1^{+/+} \mathrm{MEFs}$ were pretreated for 30 min with TAK1i ( $\mathbf{b}$ and $\mathbf{c}$ ) or SM (d) in the presence or absence of BHA (b and $\mathbf{d}$ ) or NAC (c). Cells were then stimulated by hTNF for the indicated time and cell lysates were analyzed by immunoblotting. (e) Immortalized Ripk $1^{+/+}$MEFs were pre-treated with the indicated compounds for 30 min and then stimulated with $\mathrm{hTNF}$ for $2 \mathrm{~h}$. TNFR1 complex Ilb was isolated by caspase- 8 immunoprecipitation and analyzed by immunoblotting. (f) Immortalized Ripk $1^{+/+}$MEFs were pre-treated or not with BHA for 30 min before stimulation with FLAG-hTNF for 5 min. TNFR1 complex I was analyzed by immunoprecipitation using FLAG beads followed by immunoblotting. (g) Immortalized Ripk3 ${ }^{+/+}$and Ripk3 ${ }^{-/-}$MEFs were left unstimulated or pre-treated for 30 min with TAK $1 \mathrm{i}$ in the presence or absence of zVAD-fmk and subsequently stimulated with hTNF for $3 \mathrm{~h}$. DHR-123 was added for the last $30 \mathrm{~min}$ of incubation at $37^{\circ} \mathrm{C}$. Cellular ROS in PI-negative cells was monitored using the BD LSRII flow cytometer. The results are normalized to the percentage of DHR123 + $/ \mathrm{PI}$ - cells detected in unstimulated Ripk $3^{+/+}$MEFs. Error bars indicate standard deviation from triplicate samples. The results are representative of two independent experiments. (h) Immortalized Ripk3 ${ }^{+/+}$and Ripk3 ${ }^{-/-}$MEFs were pre-treated with TAK1i in the presence or absence of zVAD-fmk, followed by stimulation with hTNF for $2 \mathrm{~h}$. TNFR1 complex llb assembly was analyzed by immunoprecipitation of caspase-8

repression only provided protection to TNF-mediated death in conditions affecting RIPK1 ubiquitylation in complex I, and not upon TAK1 kinase inhibition, supporting a role for TAK1 downstream of RIPK1 ubiquitylation in this early NF- $\kappa$ Bindependent cell death checkpoint. Further investigation is needed to understand how TAK1 regulates the death potential of RIPK1, but it is tempting to speculate that TAK1, either directly or indirectly, negatively regulates RIPK1 kinase activity at the level of complex I. Indeed, we found that TNF-mediated RIPK1-dependent apoptosis relies on RIPK1 activity, and previously reported that the sensitization of L929 cells to TNF-induced necroptosis upon TAK1 kinase inhibition was associated with enhanced RIPK1 kinase activity. ${ }^{40}$ Interestingly, it was recently reported that NEMO also negatively regulates TNF-mediated RIPK1-dependent apoptosis and necroptosis independently of NF- $\kappa$ B ${ }^{43,44}$ Whether TAK1 and NEMO collaborate in the early NF- $k$ B-independent cell death checkpoint downstream of TNFR1 by regulating RIPK1 kinase activity is an interesting possibility that will deserve future attention.

The interest for necroptosis has grown dramatically over the past few years, mainly due to recent studies demonstrating its importance under physiological and pathological conditions. ${ }^{24}$ Although the molecular mechanisms regulating necroptosis are not yet fully understood, it is well established that the kinase activities of both RIPK1 and RIPK3, and the physical interaction between these two kinases, are crucial steps in mediating this type of cell death. As a consequence, the inhibition of RIPK1 kinase activity by Nec-1 and the repression of RIPK3 levels, either by genetically or by RNA interference, have become commonly used approaches to estimate the contribution of necroptosis both in vitro and in vivo. ${ }^{29,45}$ The results presented in this study, however, demonstrate that none of these two approaches is an absolute tool to characterize necroptosis, as both RIPK1 kinase activity and RIPK3 are also required for TNF-mediated apoptosis under TNF + SM or TNF + TAK1i treatment. In line with this, our results demonstrate that ROS generation is not only required for TNF-mediated necroptosis, ${ }^{40}$ as in this study ROS scavenging also inhibited caspase activation and complex IIb assembly upon TNF stimulation. The fact that RIPK3 deficiency affects the extent of caspase-8 activation and ROS levels without disturbing complex llb assembly itself suggests the involvement of ROS both upstream and downstream of complex Illb, with RIPK3 only contributing to ROS generation downstream of complex Ilb. The idea that RIPK3 contributes to apoptosis independently of RIPK1 binding is supported by the results from our reconstitution experiment, in which both WT RIPK3 and the RHIM mutant similarly rescued caspase activation upon TNF + SM or TNF + TAK1i treatment. In addition, a RIPK1-independent role of RIPK3 in ROS-mediated caspase-8 activation has also recently been reported for inflammasome activation. ${ }^{39}$ In the context of necroptosis, two recent studies provided a potential link 


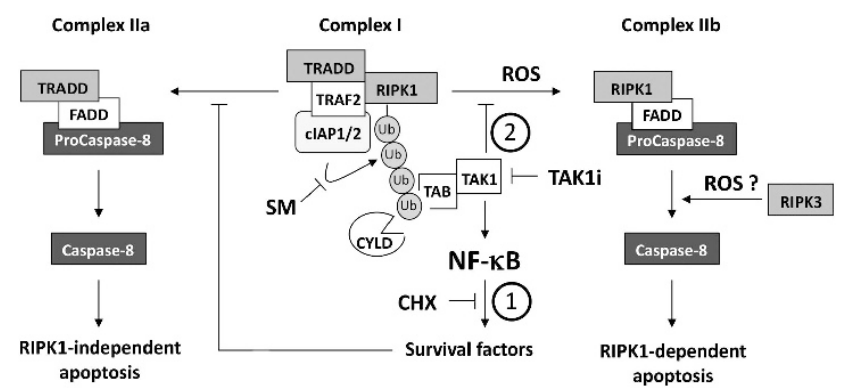

Figure 8 Model for the two cell death checkpoints in TNF-induced apoptosis. TNF stimulation leads to the assembly of the plasma membrane TNFR1 complex I. Within this complex, RIPK1 is rapidly conjugated with $\mathrm{Ub}$ chains. These $\mathrm{Ub}$ chains act as scaffolds for the recruitment and activation of the TAB2/3-TAK1 complex and the IKK complex, which subsequently leads to the activation of the canonical NF- $\kappa B$ signaling pathways that drive transcription of genes that prevent cell death - the first cell death checkpoint (1). Accordingly, when the NF- $\kappa$ B response is inhibited, either genetically or by the use of the general translation inhibitor cycloheximide (CHX), TNFR1 ligation switches from a pro-survival to a RIPK1-independent pro-apoptotic response by assembly of the cytoplasmic TNFR1 complex Ila. In addition to its role in $\mathrm{NF}-\kappa \mathrm{B}$ activation, TAK1 regulates a second cell death checkpoint at the level of complex I that prevents RIPK1 from integrating the death complex (2). Indeed, when TAK1 activity in complex I is affected, either indirectly by avoiding TAK1 recruitment through the use of Smac Mimetics (SM) - which affects RIPK1 ubiquitylation - or directly by inhibiting its kinase activity (TAK1i), TNF stimulation induces RIPK1 kinase-dependent apoptosis by assembly of the cytosolic death complex IIb. Because CYLD acts upstream of TAK1 recruitment, its repression does not protect cells from apoptosis induced by TAK1 kinase inhibition. RIPK3 contributes to full caspase-8 activation by acting downstream of complex Ilb, in a kinase and intact RHIM-independent manner. ROS regulates TNF-induced RIPK1-dependent caspase activation by acting upstream of complex Ilb assembly, and possibly also downstream of complex Ilb via RIPK3

between RIPK3 and mitochondrial ROS production that involves binding of RIPK3, and not RIPK1, to MLKL. ${ }^{46,47}$ However, MLKL probably does not fulfill this function under the apoptotic conditions studied here, as the interaction between RIPK3 and MLKL was shown to depend on RIPK3 kinase activity and our results show that the KD mutant of RIPK3 also recapitulate the sensitivity to TNF-induced apoptosis. Our results on the kinase-independent role of RIPK3 in TNF-mediated apoptosis are in line with early work reporting apoptosis induction upon ectopic expression of $\mathrm{KD}$ RIPK3 mutants. ${ }^{37,48}$ It was also previously reported that RIPK3 could, directly or indirectly, interact with the prodomain of caspase-8 upon ectopic expression. ${ }^{37}$ It is therefore possible that the contribution of RIPK3 to TNF-mediated apoptosis involves another mechanism than ROS production. Further studies are therefore required to better understand the scaffolding role of RIPK3 in TNF-induced RIPK1-dependent apoptosis and to decipher the link between RIPK3, ROS and caspase-8 under these apoptotic conditions.

Our results demonstrate that, in addition to its well-established role in necroptosis, RIPK3 contributes to TNF-induced RIPK1-dependent apoptosis in a way that does not require its intact RHIM domain and its kinase activity. Our work therefore opens doors for the re-interpretation of the specific necroptotic phenotypes reported in previous studies on RIPK3. This study also highlights the need to identify new molecules, or molecular mechanisms, to specifically interfere with necroptosis induction. Importantly, as RIPK3 kinase activity and the RHIM domain are required for necroptosis but dispensable for apoptosis induced by $\mathrm{TNF}+\mathrm{SM}$ and TNF + TAKi, the development of specific RIPK3 kinase inhibitors or small molecules that prevent homotypic RHIM domain interactions remain an interesting future challenge to specifically inhibit necroptosis. In line with this, the development of a RIPK3 kinase-dead knock-in mouse line will be of great value to estimate the relative contribution of RIPK3-dependent necroptosis versus apoptosis in physiological and pathological conditions.

\section{Materials and Methods}

Plasmids. The sequences encoding WT Ripk3 and the mutated versions of Ripk3 were cloned into pENTR3C using the cloneEZ pcr cloning kit (GenScript, Piscataway, NJ, USA). Next, these sequences were transferred into a modified pLenti6-V5-puromycin destination vector using the LR gateway recombination system (Life Technologies, Carlsbad, CA, USA). The KD contains the K51A substitution. The RHIM domain mutant was generated by mutating the four consecutive amino acids QIGN (449-451) into AAAA.

Cell lines. SV40 large T-immortalized Ripk $1^{+/+}, R i p k 1^{-/-}, R i p k 3^{+/+}$and Ripk3 ${ }^{-/-}$MEFs have previously been described. ${ }^{49}$ Primary Ripk $3^{+/+}$ and Ripk $3^{-1-}$ MEFs were isolated from E12.5 littermate embryos following standard protocol. The Tak1 $1^{+/+}$and Tak1 $1^{-1-}$ MEFs were kindly provided by Dr. Takeuchi. ${ }^{30}$ The MEFs and HEK293T cells were cultured in Dulbecco's modified Eagle's medium supplemented with $10 \%$ fetal calf serum, penicillin $(100 \mathrm{IU} / \mathrm{ml})$, streptomycin $(0.1 \mathrm{mg} / \mathrm{ml})$, L-glutamine $(200 \mu \mathrm{M})$ and sodium pyruvate $(400 \mu \mathrm{M})$. Reconstitution of the Ripk3 $3^{-1-}$ MEFs was done by lentiviral transduction. $1 \times 10^{6}$ HEK293T cells were transfected using calcium phosphate with the empty pLenti6-V5-puromycin plasmid or with the plasmids containing the different versions of Ripk3 in combination with pCAGGS-CrmA and the lentiviral packaging vectors $\mathrm{pMD2}-\mathrm{VSVG}$ and $\mathrm{pCMV}-\Delta \mathrm{R} 8.91$. The medium was changed after $6 \mathrm{~h}$, and sample was collected $48 \mathrm{~h}$ post transfection. The virus-containing supernatant was then used to infect the Ripk $3^{-1-}$ MEFs. The infected MEFs were then selected for 4 days by adding $2.5 \mu \mathrm{g} / \mathrm{ml}$ puromycin to the medium. Control retroviral pLXSN vector and $\mathrm{pLXSN}-\mathrm{I}_{\kappa} \mathrm{B} \alpha \mathrm{SR}$ vector were used to infect immortalized wt MEFs. Stable control and $\mathrm{I}_{\kappa} \mathrm{B} \alpha \mathrm{SR}$-expressing cells were obtained through selection with $\mathrm{G} 418$ at $0.5 \mu \mathrm{g} / \mathrm{ml}$ for 1 week.

Antibodies, cytokines and reagents. Antibodies were purchased from the following companies:

Anti-RIP1 (BD Biosciences, San Jose, CA, USA, \#610459), anti-RIP3 (SigmaAldrich, St Louis, MO, USA, \#R4277), anti-caspase-8 (Enzo Life Sciences Inc., Farmingdale, NY, USA, \#ALX-804-447), anti-caspase-8 (Cell Signaling, Danvers, MA, USA, \#9429), anti-caspase 3 (Cell Signaling, \#9662), anti- $\beta$ tubulin (Abcam, Cambridge, UK, \#ab6046-200), anti-cleaved PARP (Asp214; Cell Signaling, \#9544S). The anti-clAP1 antibody was a kind gift from Professor Silke (University of Melbourne). ${ }^{34}$ Recombinant human TNF- $\alpha$, produced and purified to at least $99 \%$ homogeneity in our laboratory, has a specific biological activity of $3 \times 10^{7} \mathrm{IU} / \mathrm{mg}$ and was used at $600 \mathrm{IU} / \mathrm{ml}(20 \mathrm{ng} / \mathrm{ml})$ to stimulate MEFs. Recombinant FLAG-hTNF- $\alpha$ was purchased from VIB Protein Service Facility (Ghent, Belgium) and was used at $1.5 \mu \mathrm{g} / \mathrm{ml}$. The general protein translation inhibitor CHX (Sigma-Aldrich) was used as $0.25 \mu \mathrm{g} / \mathrm{ml}$ (Figure 1c) and $5 \mu \mathrm{g} / \mathrm{ml}$ (Figure 3a). Nec-1 (Calbiochem, Merck KGaA, Darmstadt, Germany) was used at $10 \mu \mathrm{M}$. The TAK1 kinase inhibitor, NP-009245 (AnalytiCon Discovery GmbH, Potsdam, Germany), a derivative of (5Z)-7-Oxozeaenol, was used at $1 \mu \mathrm{M}$. The caspase peptide inhibitor, zVAD-fmk (Bachem, Bubendorf, Switzerland), was used at $20 \mu \mathrm{M}$. Compound A (TetraLogic Pharmaceuticals Inc., Malvern, PA, USA) was used at $500 \mathrm{nM}$. BHA (Sigma-Aldrich) was used at $100 \mu \mathrm{M}$. $N$-Acetyl-L-cysteine (Sigma-Aldrich) was used at $5 \mathrm{mM}$.

Analysis of cell death and caspase-3 activity using the Fluostar Omega fluorescence plate reader (BMG Labtech GmbH, Ortenberg, Germany). MEFs were seeded at least in triplicate at 7500 cells per well in a 96-well adherent plate using L15 medium (Leibovitz medium) or DMEM. The next day, the cells were pre-treated with the indicated compounds for $30 \mathrm{~min}$ and then stimulated with hTNF $(600 \mathrm{IU} / \mathrm{ml})$ in the presence of $5 \mu \mathrm{M}$ SytoxGreen (Life Technologies) and $20 \mu \mathrm{M}$ DEVD-AMC (PeptaNova GmbH, Sandhausen, Germany). SytoxGreen and DEVD-AMC fluorescence intensity were measured in 
function of the time at intervals of $1 \mathrm{~h}$ by using a Fluostar Omega fluorescence plate reader, with excitation/emission filters of $485 / 520 \mathrm{~nm}$ for SytoxGreen and $360 / 460 \mathrm{~nm}$ for DEVD-AMC. The gains set at 1100, 20 flashes per well were taken, with orbital averaging with a diameter of $3 \mathrm{~mm}$. Cell death was calculated by subtracting the induced SytoxGreen fluorescence from the background fluorescence and by dividing the obtained result by the maximal fluorescence (minus the background fluorescence) obtained by permeabilization of the cells using Triton $\mathrm{X}-100$ at a final concentration of $0.1 \%$. The caspase- 3 activity was calculated by subtracting the induced DEVD-AMC fluorescence from the background fluorescence and by dividing the obtained result with the SytoxGreen fluorescence obtained from the Triton X-100 permeabilized cells, which served to normalized the value to the amount of cells seeded. All experiments were performed at least twice in triplicates. Validation of the system is presented in Supplementary Figure S3.

Intracellular ROS measurement. MEFs were seeded in triplicate at $3 \times 10^{5}$ cells/well (six-well plate). The next day, the cells were pre-treated with TAK 1 kinase inhibitor for $30 \mathrm{~min}$ and stimulated with $\mathrm{hTNF}$ for an additional $3 \mathrm{~h}$. In the last $30 \mathrm{~min}$ of incubation at $37^{\circ} \mathrm{C}$, the cells were supplemented with dihydrorodamine 123 (DHR123). Following treatment, the cells were trypsinized and re-suspended in $1 \mathrm{ml}$ of PI-containing medium. Cellular ROS production was determined by measuring the conversion of DHR123 to R123 by flow cytometry on a triple-laser $(405,488$ and $635 \mathrm{~nm})$ LSR-II using the FACSDiva software (BD Biosciences). Only viable cells (PI negative) were gated for the analysis of ROS production. ROS measurement was performed twice in triplicate.

Immunoprecipitation. MEFs were seeded at $2 \times 10^{6} \mathrm{cells} / 10 \mathrm{~cm}$ plate the day before stimulation. After treatment with the indicated triggers, MEFs were washed with PBS and lysed in NP-40 lysis buffer $(150 \mathrm{mM} \mathrm{NaCl}, 1 \% \mathrm{NP}-40,10 \%$ glycerol, $10 \mathrm{mM}$ Tris-HCl pH 8) supplemented with EDTA-free protease inhibitor cocktail tablets (Roche Diagnostics, Basel, Switzerland, \#11873580001) and phosphatase inhibitor cocktail tablets (Roche Diagnostics, \#04906837001). For TNFR1 complex I immunoprecipitation (5 min post stimulation), TNFRI bound to human recombinant FLAG-hTNF was immunoprecipitated overnight using anti-FLAG M2 affinity gel (Sigma-Aldrich). For TNFR1 complex II immunoprecipitation (2h post stimulation), endogenous caspase-8 was immunoprecipitated overnight using anticaspase-8-coupled protein A beads. For immunoprecipitation of processed caspase8, MEFs were first pre-treated with biotin-IETD-fmk (MBL International, Woburn, MA, USA, \#JM-1121-20C) for $30 \mathrm{~min}$ and then stimulated with hTNF and SM for an additional $3.5 \mathrm{~h}$. The cells were then washed with PBS, lysed and processed caspase-8 bound to biotin-IETD-fmk was immunoprecipitated overnight using streptavidin beads (Life Technologies, \#S59G). After all immunoprecipitations, the beads were washed three times in NP-40 lysis buffer and the immunoprecipitated proteins were eluted by adding $60 \mu \mathrm{l}$ of $2 \times$ Laemmli buffer to the beads. Complexes were subsequently analyzed by immunoblotting (20 $\mu$ l/lane)

RNAi-mediated knockdown. $1 \times 10^{6} \mathrm{MEF}$ cells were seeded in a $75-\mathrm{cm}^{2}$ flask and transfected the next day according to the manufacturer's protocol using $25 \mathrm{nM}$ siRNA-targeting CYLD or $25 \mathrm{nM}$ siCONTROL non-targeting siRNA (ON-TARGETplus SMART pool siRNA; Dharmacon, Thermo Fisher Scientific, Waltham, MA, USA). After $48 \mathrm{~h}$, the cells were trypsinized and seeded. The next day, the cells were stimulated with hTNF and cell death or recruitment of RIPK1 to caspase-8 was determined as described above. Knockdown efficiency was tested by immunoblotting. To achieve a stable miRNA-mediated knockdown of RIPK1 and RIPK3 in the immortalized Ripk1 $1^{+/+}$MEFs, oligos against the $3^{\prime}$ UTR of RIPK1 and RIPK3 were cloned into BLOCK-iT HiPerform Lentiviral Pol II miR RNAi Expression System with EmGFP (Life Technologies) and subsequently cloned into the pLenti6.2-V5 destination vector (Life Technologies) by the gateway system. These miRNA-containing plasmids were introduced into immortalized Ripk $1^{+/+}$MEFs by lentiviral transduction as described above.

\section{Conflict of Interest}

The authors declare no conflict of interest.

Acknowledgements. We thank Dr. Nozomi Takahashi for constructive scientific discussion. We are also grateful to Kim Newton and Vishva Dixit for providing us with the RIPK3 knockout mouse line that was used to generate Ripk3 ${ }^{+1+}$ and Ripk3 ${ }^{-1-}$ MEFs, and TetraLogic Pharmaceuticals, Inc. for providing us with the SM CmpA. Y.D. is holder of a Ph.D. fellowship from the Agency for Innovation by Science and Technology (IWT). M.J.M.B. has a tenure track position in the Multidisciplinary Research Program of Ghent University (GROUP-ID). Research in his group is supported by grants from the Research Foundation Flanders (FWO G.0172.12N) and Interuniversity Attraction Poles (IAP 7). Research in the Vandenabeele group is supported by the European grants (Euregional PACT II), Belgian grants (Interuniversity Attraction Poles, IAP 7/32), Flemish grants (Research Foundation Flanders - FWO G.0875.11, FWO G.0973.11, FWO G.0A45.12N, Methusalem grant - BOF09/01M00709), Ghent University grants (MRP, GROUP-ID consortium), grant from the Foundation against Cancer, F94 and grants from VIB. Research in the Dejardin group is supported by Belgian grants from the Interuniversity Attraction Poles, (IAP 7/32) and the « Fédération belge Contre le Cancer ».

1. Varfolomeev EE, Ashkenazi A. Tumor necrosis factor: an apoptosis JuNKie? Cell 2004; 116: 491-497.

2. Vandenabeele P, Declercq W, Vanhaesebroeck B, Grooten J, Fiers W. Both TNF receptors are required for TNF-mediated induction of apoptosis in PC60 cells. $\mathrm{J}$ Immunol 1995; 154: 2904-2913

3. Wilson NS, Dixit V, Ashkenazi A. Death receptor signal transducers: nodes of coordination in immune signaling networks. Nat Immunol 2009; 10: 348-355.

4. Vandenabeele P, Declercq W, Van Herreweghe F, Vanden Berghe T. The role of the kinases RIP1 and RIP3 in TNF-induced necrosis. Sci Signal 2010; 3: re4.

5. Micheau O, Tschopp J. Induction of TNF receptor I-mediated apoptosis via two sequential signaling complexes. Cell 2003; 114: 181-190.

6. Walczak $H$. TNF and ubiquitin at the crossroads of gene activation, cell death, inflammation, and cancer. Immunol Rev 2011; 244: 9-28.

7. Gerlach B, Cordier SM, Schmukle AC, Emmerich CH, Rieser E, Haas TL et al. Linear ubiquitination prevents inflammation and regulates immune signalling. Nature 2011; 471: 591-596

8. Dynek JN, Goncharov T, Dueber EC, Fedorova AV, Izrael-Tomasevic A, Phu L et al. c-IAP1 and UbcH5 promote K11-linked polyubiquitination of RIP1 in TNF signalling. EMBO J 2010; 29: 4198-4209.

9. Newton K, Matsumoto ML, Wertz IE, Kirkpatrick DS, Lill JR, Tan J et al. Ubiquitin chain editing revealed by polyubiquitin linkage-specific antibodies. Cell 2008; 134: 668-678

10. Mahoney DJ, Cheung HH, Mrad RL, Plenchette S, Simard C, Enwere E et al. Both clAP1 and clAP2 regulate TNFalpha-mediated NF-kappaB activation. Proc Natl Acad Sci USA 2008; 105: 11778-11783.

11. Declercq W, Vanden Berghe T, Vandenabeele P. RIP kinases at the crossroads of cell death and survival. Cell 2009; 138: 229-232.

12. Wang $L$, Du F, Wang $X$. TNF-alpha induces two distinct caspase-8 activation pathways. Cell 2008; 133: 693-703.

13. Gentle IE, Wong WW, Evans JM, Bankovacki A, Cook WD, Khan NR et al. In TNF-stimulated cells, RIPK1 promotes cell survival by stabilizing TRAF2 and cIAP1, which limits induction of non-canonical NF-kappaB and activation of caspase-8. J Biol Chem 2011; 286: 13282-13291.

14. Varfolomeev E, Goncharov T, Fedorova AV, Dynek JN, Zobel K, Deshayes K et al. c-IAP1 and C-IAP2 are critical mediators of tumor necrosis factor alpha (TNFalpha)-induced NF-kappaB activation. J Biol Chem 2008; 283: 24295-24299.

15. Bertrand MJ, Milutinovic S, Dickson KM, Ho WC, Boudreault A, Durkin J et al. clAP1 and cIAP2 facilitate cancer cell survival by functioning as E3 ligases that promote RIP1 ubiquitination. Mol Cell 2008; 30: 689-700.

16. Moulin M, Anderton H, Voss AK, Thomas T, Wong WW, Bankovacki A et al. IAPs limit activation of RIP kinases by TNF receptor 1 during development. EMBO $\mathrm{J} 2012 ; 31$ : 1679-1691.

17. Petersen SL, Wang L, Yalcin-Chin A, Li L, Peyton M, Minna J et al. Autocrine TNFalpha signaling renders human cancer cells susceptible to Smac-mimetic-induced apoptosis. Cancer Cell 2007; 12: 445-456.

18. Wong WW, Gentle IE, Nachbur U, Anderton H, Vaux DL, Silke J. RIPK1 is not essential for TNFR1-induced activation of NF-kappaB. Cell Death Differ 2010; 17: 482-487.

19. Park SM, Yoon JB, Lee TH. Receptor interacting protein is ubiquitinated by cellular inhibitor of apoptosis proteins (C-IAP1 and C-IAP2) in vitro. FEBS Lett 2004; 566: 151-156.

20. Haas TL, Emmerich $\mathrm{CH}$ Gerlach B Schmukle AC, Cordier SM, Rieser E et al. Recruitment of the linear ubiquitin chain assembly complex stabilizes the TNF-R1 signaling complex and is required for TNF-mediated gene induction. Mol Cell 2009; 36: 831-844.

21. Vince JE, Pantaki D, Feltham R, Mace PD, Cordier SM, Schmukle AC et al. TRAF2 must bind to cellular inhibitors of apoptosis for tumor necrosis factor (tnf) to efficiently activate nf-\{kappa\}b and to prevent tnf-induced apoptosis. J Biol Chem 2009; 284: 35906-35915.

22. O'Donnell MA, Legarda-Addison D, Skountzos $P$, Yeh WC, Ting AT. Ubiquitination of RIP1 regulates an NF-kappaB-independent cell-death switch in TNF signaling. Curr Biol 2007; 17: 418-424.

23. Vucic D, Dixit VM, Wertz IE. Ubiquitylation in apoptosis: a post-translational modification at the edge of life and death. Nat Rev. Mol Cell Biol 2011; 12: 439-452. 
24. Vandenabeele P, Galluzzi L, Vanden Berghe T, Kroemer G. Molecular mechanisms of necroptosis: an ordered cellular explosion. Nat Rev. Mol Cell Biol 2010; 11: 700-714.

25. He S, Wang L, Miao L, Wang T, Du F, Zhao L et al. Receptor interacting protein kinase-3 determines cellular necrotic response to TNF-alpha. Cell 2009; 137: 1100-1111.

26. Cho YS, Challa S, Moquin D, Genga R, Ray TD, Guildford M et al. Phosphorylation-driven assembly of the RIP1-RIP3 complex regulates programmed necrosis and virus-induced inflammation. Cell 2009; 137: 1112-1123.

27. Zhang DW, Shao J, Lin J, Zhang N, Lu BJ, Lin SC et al. RIP3, an energy metabolism regulator that switches TNF-induced cell death from apoptosis to necrosis. Science 2009; 325: 332-336.

28. Degterev A, Hitomi J, Germscheid M, Ch'en IL, Korkina O, Teng X et al. Identification of RIP1 kinase as a specific cellular target of necrostatins. Nat Chem Biol 2008; 4: 313-321.

29. Galluzzi L, Vitale I, Abrams JM, Alnemri ES, Baehrecke EH, Blagosklonny MV et al. Molecular definitions of cell death subroutines: recommendations of the Nomenclature Committee on Cell Death 2012. Cell Death Differ 2012; 19: 107-120.

30. Sato S, Sanjo H, Takeda K, Ninomiya-Tsuji J, Yamamoto M, Kawai T et al. Essential function for the kinase TAK1 in innate and adaptive immune responses. Nat Immunol 2005; 6: 1087-1095.

31. Shim JH, Xiao C, Paschal AE, Bailey ST, Rao P, Hayden MS et al. TAK1, but not TAB1 or TAB2, plays an essential role in multiple signaling pathways in vivo. Genes Dev 2005; 19 : 2668-2681.

32. Thiefes A, Wolter S, Mushinski JF, Hoffmann E, Dittrich-Breiholz O, Graue N et al. Simultaneous blockade of NFkappaB, JNK, and p38 MAPK by a kinase-inactive mutant of the protein kinase TAK1 sensitizes cells to apoptosis and affects a distinct spectrum of tumor necrosis factor [corrected] target genes. J Biol Chem 2005; 280: 27728-27741.

33. Arslan SC, Scheidereit C. The prevalence of TNFalpha-induced necrosis over apoptosis is determined by TAK1-RIP1 interplay. PloS One 2011; 6: e26069.

34. Vince JE, Wong WW, Khan N, Feltham R, Chau D, Ahmed AU et al. IAP antagonists target clAP1 to induce TNFalpha-dependent apoptosis. Cell 2007; 131: 682-693.

35. Brown K, Gerstberger S, Carlson L, Franzoso G, Siebenlist U. Control of I kappa B-alpha proteolysis by site-specific, signal-induced phosphorylation. Science 1995; 267: 1485-1488.

36. Geserick P, Hupe M, Moulin M, Wong WW, Feoktistova M, Kellert B et al. Cellular IAPs inhibit a cryptic CD95-induced cell death by limiting RIP1 kinase recruitment. J Cell Biol 2009; 187: 1037-1054.
37. Sun X, Lee J, Navas T, Baldwin DT, Stewart TA, Dixit VM. RIP3, a novel apoptosisinducing kinase. J Biol Chem 1999; 274: 16871-16875.

38. Yu PW, Huang BC, Shen M, Quast J, Chan E, Xu X et al. Identification of RIP3, a RIP-like kinase that activates apoptosis and NFkappaB. Curr Biol 1999; 9: 539-542.

39. Vince JE, Wong WW, Gentle I, Lawlor KE, Allam R, O'Reilly L et al. Inhibitor of apoptosis proteins limit RIP3 kinase-dependent interleukin-1 activation. Immunity 2012; 36: 215-227.

40. Vanlangenakker N, Vanden Berghe T, Bogaert P, Laukens B, Zobel K, Deshayes $\mathrm{K}$ et al. CIAP1 and TAK1 protect cells from TNF-induced necrosis by preventing RIP1/RIP3dependent reactive oxygen species production. Cell Death Differ 2011; 18: 656-665.

41. Ikner A, Ashkenazi A. TWEAK induces apoptosis through a death-signaling complex comprising receptor-interacting protein 1 (RIP1), Fas-associated death domain (FADD), and caspase-8. J Biol Chem 2011; 286: 21546-21554.

42. Ea CK, Deng L, Xia ZP, Pineda G, Chen ZJ. Activation of IKK by TNFalpha requires site-specific ubiquitination of RIP1 and polyubiquitin binding by NEMO. Mol Cell 2006; 22 : 245-257.

43. O'Donnell MA Hase $H$, Legarda $D$, Ting AT. NEMO inhibits programmed necrosis in an NFkappaB-independent manner by restraining RIP1. PLoS One 2012; 7: e41238.

44. Legarda-Addison $\mathrm{D}$, Hase $\mathrm{H}, \mathrm{O}$ 'Donnell MA, Ting AT. NEMO/IKKgamma regulates an early NF-kappaB-independent cell-death checkpoint during TNF signaling. Cell Death Differ 2009; 16: 1279-1288.

45. Duprez L, Takahashi N, Van Hauwermeiren F, Vandendriessche B, Goossens V, Vanden Berghe T et al. RIP kinase-dependent necrosis drives lethal systemic inflammatory response syndrome. Immunity 2011; 35: 908-918.

46. Sun L, Wang H, Wang Z, He S, Chen S, Liao D et al. Mixed lineage kinase domain-like protein mediates necrosis signaling downstream of RIP3 kinase. Cell 2012; 148: 213-227.

47. Zhao J, Jitkaew S, Cai Z, Choksi S, Li Q, Luo J et al. Mixed lineage kinase domain-like is key receptor interacting protein 3 downstream component of TNF-induced necrosis. Proc Natl Acad Sci USA 2012; 109: 5322-5327.

48. Kasof GM, Prosser JC, Liu D, Lorenzi MV, Gomes BC. The RIP-like kinase, RIP3, induces apoptosis and NF-kappaB nuclear translocation and localizes to mitochondria. FEBS Lett 2000; 473: 285-291.

49. Jouan-Lanhouet S, Arshad MI, Piquet-Pellorce C, Martin-Chouly C, Le Moigne-Muller G, Van Herreweghe $F$ et al. TRAIL induces necroptosis involving RIPK1/RIPK3-dependent PARP-1 activation. Cell Death Differ 2012; 19: 2003-2014.

\section{Supplementary Information accompanies this paper on Cell Death and Differentiation website (http://www.nature.com/cdd)}

